\title{
基于摆线刀齿轨迹的未变形铣屑厚度分析*
}

\author{
荬 炜 ${ }^{1}$ 崔岗卫 ${ }^{2}$ 袁胜万 $^{2}$ 何晓聪 ${ }^{1}$ \\ (1. 昆明理工大学机电工程学院 昆明 650500; \\ 2. 沈机集团昆明机床股份有限公司 昆明 650203)
}

\begin{abstract}
摘要: 利用动态变化的未变形铣屑厚度与铣削力之间的相互作用关系, 研究铣削过程的动态特性是铣削过程建模的基本思路。 未变形铣屑厚度的值取决于相继切入工件的刀刃在工件上留下的切削痕迹之间的相对位置关系。处于切削状态的刀刃与前一 齿尖所经过的摆线运动轨迹相交, 以其方位角与前一刀齿过同一交点时的方位角之差为辅助变量, 建立满足铣屑形成条件的 运动学超越方程。若该辅助变量已知, 则可进一步求出铣削迟滞参数及未变形铣屑厚度。基于这一思路, 给出两种新的求取 铣屑厚度的方法: 一种是将原方程转化为解描述辅助变量动态变化的微分方程, 采用数值方法求得其数值解; 另一种是以辅 助变量的线性函数近似原方程中的三角函数, 采用近似解析法解出其显式表达式。仿真结果表明: 对于当前制造业普遍采用的 铁削参数, 所提近似解析法可以满足实际应用的精度要求, 并且与现有摆线铣屑厚度模型相比数学表达式更为简洁; 所提数值 法不需要循环迭代求解超越方程, 非常适合嵌入到对分析精度和运算效率有较高要求的铁削过程仿真或稳定性预测算法。

关键词: 铣削过程; 摆线刀齿轨迹; 未变形切屑厚度; 时变迟滞; 数值方法; 近似解析解

中图分类号: TG161; TG501
\end{abstract}

\section{Analysis of Uncut Milling Chip Thickness Based on Trochoidal Tooth Path}

\author{
DOU Wei ${ }^{1}$ CUI Gangwei ${ }^{2} \quad$ YUAN Shengwan $^{2} \quad$ HE Xiaocong $^{1}$
}

(1. Faculty of Mechanical and Electrical Engineering, Kunming University of Science and Technology, Kunming 650500;

2. Shenji Group Kunming Machine Tool Company Limited, Kunming 650203)

\begin{abstract}
One of the key issues in the modeling of milling processes is to determine the response of the cutting forces to the dynamic variations of the uncut chip thickness. The uncut chip thickness depends on the relative position between the milled surfaces left by the successive cutting teeth of the tool. A cutting tooth intersects the path of its previous tooth, and forms an angle with the previous tooth passing through the same point of intersection. Given the intersection angle, both the variable time delay and the instantaneous uncut chip thickness can be calculated directly. Through the analysis of trochoidal tooth paths and using the intersection angle as an auxiliary variable, a transcendental equation is developed to model of the geometry of chip formation. Then two new approaches are proposed to determine the intersection angle. The first approach converts the transcendental equation into an ordinary differential equation of the intersection angle, then solving it numerically without recursive root-finding algorithms; assuming the intersection angle is infinitesimal, another approach approximates the transcendental equation by replacing the sine function with a liner function, and then solve it analytically. Case studies with different process parameters show that the analytical approach can provide a high accuracy in practical milling operations, with a simpler expression compared with other models. The proposed numerical method is suitable for embedding into the milling process simulation or stability prediction algorithms which are sensitive to accuracy and efficiency.
\end{abstract}

Key words: milling processes; trochoidal tooth path; uncut chip thickness; variable delay; numeric method; approximate analytic solution

\section{0 前言}

在铣削过程中, 铣刀各齿周期性的切入和切出

* 国家科技重大专项资助项目(2016ZX04004002)。20180411 收到初稿, 20180926 收到修改稿
工件, 产生大小和方向不断变化的动态铣削力，迫 使工件和刀具发生受迫振动。振动一方面使刀具在 工件加工表面留下振纹, 影响表面粗粘度, 另一方 面导致未变形铣屑厚度发生动态变化。未变形铣屑 厚度与铣削力相互起着 “调制” 作用, 在一定不利 条件下可能导致铣削力发展为不稳定的激振力, 进 而引起整个铣削系统失稳, 形成所谓的 “颤振” 。 
为了在无颤振条件下, 尽可能高效率地获得高质量 的加工表面, 需要精确地预测动态铣削力, 以将铣 削系统的受迫振动状态控制在可以接受的范围内。

MARTELLOTTI 是最早对铣削过程进行详细研 究的学者之一, 他主张从数学的角度来研究铣削过 程 ${ }^{[1]}$ 。MARTELLOTTI 指出铣削过程中刀齿的运动 轨迹是摆线, 平均铣削力测试数据与未变形铣削厚 度之间存在线性关系, 并推导出未变形铣屑厚度的 近似解析表达式为

$$
h_{j}=R+f_{\mathrm{z}} \sin \phi_{j}-\sqrt{R^{2}-f_{\mathrm{z}}^{2} \cos ^{2} \phi_{j}}
$$

式中, 下标 $j$ 对应铣刀的第 $j$ 个刀齿; $R$ 表示铣刀半 径; $f_{z}$ 表示每齿进给量; $\phi_{j}$ 表示第 $j$ 齿的方位角。当 $f_{\mathrm{z}}$ 远小于 $R$ 时，式(1)可简化为

$$
h_{j}=f_{\mathrm{z}} \sin \phi_{j}
$$

若在推导未变形铣屑厚度的过程中, 假设刀齿的运 动轨迹是圆形, 也可以得到式(2)的结果, 因此式(2) 通常被称为 “圆形铣屑模型” ，而将式(1)称为 “摆 线铣屑模型”。SRIDHAR 等 ${ }^{[2]}$ 根据再生振动理论指 出, MARTELLOTTI的模型是名义未变形铣屑厚度, 体现的是铣削进给运动导致的铣屑变化, 为了反映 铣削过程中刀具和工件的动态位移对未变形铣屑厚 度的影响, 实际厚度可表示为

$$
\left\{\begin{array}{l}
h_{j}(t)=h_{\mathrm{s}, j}(t)+h_{\mathrm{d}, j}(t) \\
h_{\mathrm{s}, j}(t)=f_{\mathrm{z}} \sin \phi_{j}(t) \\
h_{\mathrm{d}, j}(t)=\eta_{j}(t)-\eta_{j-1}(t-\tau)
\end{array}\right.
$$

式中, $h_{\mathrm{s}, j}$ 表示名义未变形铣屑厚度(下文简称名义厚 度); $h_{\mathrm{d}, j}$ 表示动态未变形铣屑厚度(下文简称动态厚 度); $\eta_{j}$ 表示第 $j$ 齿切削工件时, 刀具相对工件的动态 位移沿加工面法向的分量; $\tau$ 称为迟滞参数, 表示第 $j-1$ 齿生成的加工面被第 $j$ 齿切削时所间隔的时间。 从实用的角度出发, 大部分铣削情况下式(2)相对式(1) 引入的误差可以忽略 ${ }^{[3]}$, 并且在圆形刀齿轨迹的假设 下迟滞参数 $\tau$ 等于刀齿经过周期 $T$, 而匀速加工时 $T$ 为常数, 从而消除了许多铣削过程建模和分析中的数 学障碍, 因此式(3)得到广泛采用 ${ }^{[4-7]}$ 。

为了尽可能精确地分析动态铣削力对加工表面 质量的影响, MONTGOMERY 等 ${ }^{[8-9]}$ 基于摆线轨迹 构建了分析薄壁立铣过程的动力学模型。他们通过 数值积分求得刀具和工件表面在每一个时间步的位 移矢量, 两矢量之间的距离即为铣屑厚度 (除非指 明, 下文铣屑厚度均指摆线刀齿轨迹下的未变形铣屑 厚度)。CAMPOMANES 等 ${ }^{[10]}$ 基于 MONTGOMERY 的模型, 采用圆弧插值算法改善了铣屑厚度的计算
精度; SCHMITZ 等 ${ }^{[11]}$ 采用线性插值算法改善了铣 屑厚度的计算效率。在文献中 [12], LI 采用数值方 法计算处于切削状态的刀刃在时刻 $t$ 的位移矢量, 以及前一刀刃在 $t-T$ 之前的数个时间步长的位移向 量, 取两刀刃的最小距离作为铣屑厚度。

类似于 MARTELLOTTI 的思路, LI 等 ${ }^{[13]}$ 利用 泰勒级数化简刀齿运动学方程, 给出了一种新的名 义厚度的近似解析表达式。KUMANCHIK ${ }^{[14]}$ 和问雪 等 ${ }^{[15]}$ 也提出了不同的名义厚度近似表达式。LONG 根据摆线运动的规律, 在文献[16]中首次指出迟滞 参数 $\tau$ 并不是常数, 且由式(3)可知, 动态厚度受 $\tau$ 影响, 因此摆线轨迹铣屑模型将使铣削过程呈现出 不同于 “圆形轨迹” 模型的动态特性 ${ }^{[17-18]}$ 。 FAASSEN 等 ${ }^{[19]}$ 结合了 LI 等 ${ }^{[13]}$ 和 LONG 等 ${ }^{[16]}$ 的成 果, 给出了一种更简单的名义厚度表达式。

除了纯数值方法 ${ }^{[8-12]}$ 和近似解析法 ${ }^{[13-19]}$ 之外, 也可以利用牛顿-拉夫森法等迭代法求解刀齿运动 学方程得到铣削厚度 ${ }^{[20]}$, 某种程度上这可以称为半 解析-半数值法。这种方法结合了数值法精度高和解 析法简洁灵活的优点，但是需要在每一个时间步长 都进行迭代搜索。在铣削过程分析中循环迭代求解 铣屑厚度, 降低了整个分析的效率。针对这一问题, 本文给出一种新的求解刀齿运动学方程的数值方 法, 从而改善铣削过程的分析效率。同时，本文也 给出一种新的铣屑近似解析模型, 该模型同时包含 名义厚度及动态厚度。

\section{1 铣削过程中的刀齿动力学分析}

为了简明起见, 本文假设铣刀为直齿, 但所得 结论不失一般性, 也适用于螺旋齿铣刀。以四齿立 铣刀为例, 图 1 所示为铣削过程的两自由度集中质 量动力学模型。惯性坐标系 $X O Y$ 固连在铣刀中心。 铣刀以转速 $\omega$ 顺时针定轴转动的同时以进给量 $f_{\mathrm{z}}$ 沿 $X$ 轴方向平移。这两个运动合成得到图中虚线所示 的摆线运动轨迹。点 $P$ 表示第 $j$ 个齿的角位移为 $\phi_{j}$ 时的齿尖实际位置, 而点 $P^{*}$ 表示齿 $j$ 与前一刀齿加 工过的工件表面的交点。 $P$ 和 $P^{*}$ 之间的距离即为铣 屑厚度 $h_{j}$ 。图 1 所示模型对应的动力学方程为

$$
\boldsymbol{M} \ddot{\boldsymbol{q}}+\boldsymbol{C} \dot{\boldsymbol{q}}+\boldsymbol{K} \boldsymbol{q}=\sum_{j=1}^{N_{t}}\left[\begin{array}{cc}
-\cos \phi_{j} & -\sin \phi_{j} \\
\sin \phi_{j} & -\cos \phi_{j}
\end{array}\right]\left[\begin{array}{c}
F_{\mathrm{t}, j} \\
F_{\mathrm{n}, j}
\end{array}\right]
$$

式中, 矩阵 $\boldsymbol{M} 、 \boldsymbol{C}$ 和 $\boldsymbol{K}$ 分别表示铣削系统在刀尖处 的等效模态质量、模态阻尼和模态刚度; $\boldsymbol{q}=[x(t)$, $y(t)]^{\mathrm{T}}$, 表示刀具中心的动态位移; $F_{\mathrm{t}, j}$ 和 $F_{\mathrm{n}, j}$ 分别表 示第 $j$ 个刀齿的角位移为 $\phi_{j}$ 时受到的动态切向力和 
法向力。当刀齿 $j$ 切割的铣屑厚度为 $h_{j}$ 时, 所受铣 削力为 ${ }^{[6]}$

$$
\left[\begin{array}{c}
F_{\mathrm{t}, j} \\
F_{\mathrm{n}, j}
\end{array}\right]=g\left(\phi_{j}\right)\left(\left[\begin{array}{l}
K_{\mathrm{t}, \mathrm{c}} \\
K_{\mathrm{n}, \mathrm{c}}
\end{array}\right] h_{j}+\left[\begin{array}{c}
K_{\mathrm{t}, \mathrm{e}} \\
K_{\mathrm{n}, \mathrm{e}}
\end{array}\right]\right) a_{p}
$$

式中, $K_{\mathrm{t}, \mathrm{c}}$ 和 $K_{\mathrm{n}, \mathrm{c}}$ 表示切向和法向前刀面剪切系数, $K_{\mathrm{t}, \mathrm{e}}$ 和 $K_{\mathrm{n}, \mathrm{e}}$ 表示切向和法向后刀面犁切力系数; $a_{p}$ 表示轴向切入深度; 函数 $g\left(\phi_{j}\right)$ 称为切削状态窗函数, 用于判定刀齿是否处于切削状态, 当刀齿切削时其 值为 1 , 否则为 0

$$
g\left(\phi_{j}\right)= \begin{cases}1 & \phi_{\mathrm{s}} \leqslant \phi_{j}+2 k \pi \leqslant \phi_{\mathrm{e}}, k=0,1, \cdots \\ 0 & \text { 其他 }\end{cases}
$$

式中, $\phi_{\mathrm{s}}$ 和 $\phi_{\mathrm{e}}$ 分别称为切入和切出角, 其值取决于 铣刀尺寸、径向切深和铣削方式。

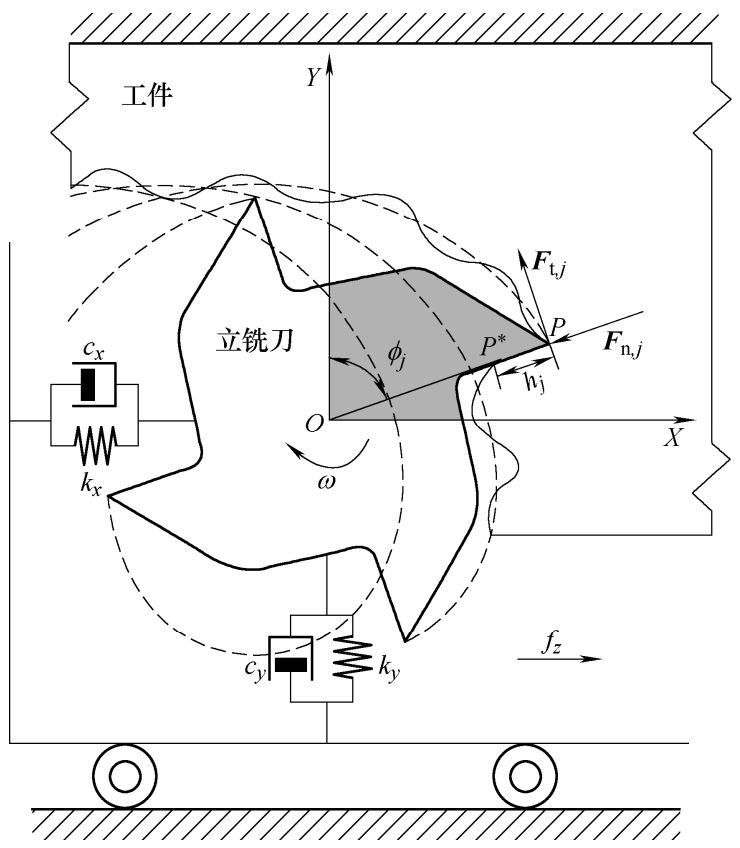

图 1 铣削过程动力学模型

如前所述, 铣削过程中的刀齿运动轨迹可以分 解为名义轨迹和动态铣削力作用下偏离名义轨迹的 部分。忽略刀具振动的情况下, 铣削运动中任意相 邻两刀齿的几何关系如图 2 所示: 点 $O$ 和 $O_{1}$ 分别 表示时刻 $t$ 和时刻 $t-\tau_{j}$ 的铣刀中心位置; 线段 $O Q_{j}$ 和 $O Q_{j-1}$ 分别代表第 $j$ 齿和第 $j-1$ 齿在 $t$ 时刻的位置, 而线段 $O_{1} Q_{0}$ 和 $O_{1} Q_{1}$ 则分别表示第 $j$ 齿和第 $j-1$ 齿 在 $t-\tau_{j}$ 时刻的位置; $\phi_{j}$ 表示表示 $O Q_{j}$ 在时刻 $t$ 的方位 角, 而 $\phi_{1}$ 表示 $O_{1} Q_{1}$ 在时刻 $t-\tau_{j}$ 的方位角。 $Q_{\mathrm{s}}$ 和 $Q_{\mathrm{e}}$ 分别表示槽铣时, 第 $j$ 齿的切入和切出点。线段 $Q_{1} Q_{j}$ 的长度即为名义厚度

$$
h_{s, j}=\left\|O Q_{j}\right\|-\left\|O Q_{1}\right\|
$$

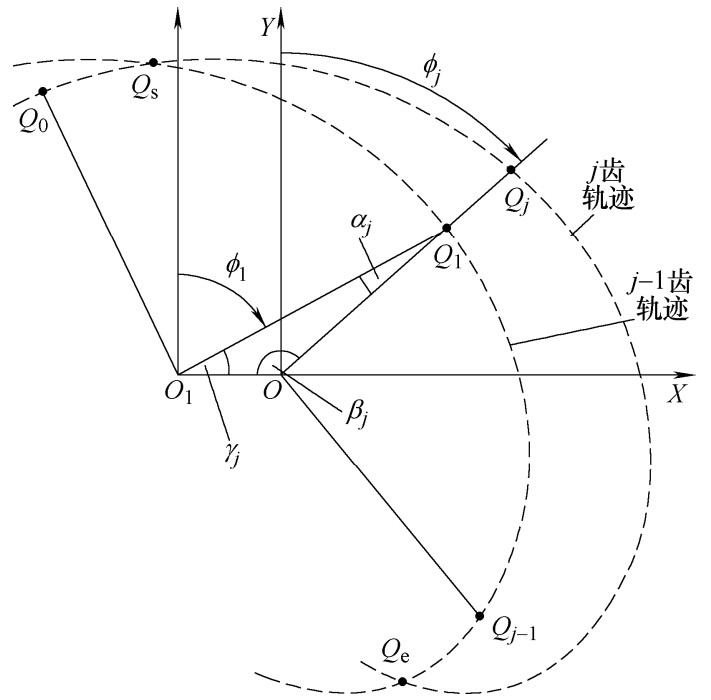

图 2 刀齿摆线运动轨迹

对任意刀齿 $j$, 只要已知铣刀中心的瞬时位置 $O$ 和方位角 $\phi_{j}$, 则齿尖在任意时刻 $t$ 的位置 $Q_{j}$ 为

$$
Q_{j}(t)=\left[\begin{array}{c}
x_{j}(t) \\
y_{j}(t)
\end{array}\right]=\left[\begin{array}{c}
f_{\mathrm{z}} \frac{t}{T}+R \sin \phi_{j}(t) \\
R \cos \phi_{j}(t)
\end{array}\right]
$$

式中, $R$ 表示铣刀半径; 刀齿通过周期 $T=2 \pi /\left(\omega N_{\mathrm{t}}\right), N_{\mathrm{t}}$ 为铣刀齿数。方位角 $\phi_{j}$ 可由下式确定

$$
\phi_{j}(t)=\omega t-\phi_{\mathrm{p}}(j-1) \quad j=1,2, \cdots, N_{\mathrm{t}}
$$

式中, $\phi_{\mathrm{p}}=2 \pi / N_{\mathrm{t}}$, 称为节距角。为了简明起见, 图 2 用 $\phi_{1}$ 表示第 $j-1$ 齿在 $t-\tau_{j}$ 时刻的方位角, 即

$$
\phi_{1}=\phi_{j-1}\left(t-\tau_{j}\right)=\phi_{j}+\phi_{\mathrm{p}}\left(1-\frac{\tau_{j}}{T}\right)
$$

根据式(8)和 $\triangle O Q_{1} O_{1}$ 可得如下关系

$$
\frac{\left\|O O_{1}\right\|}{\sin \alpha_{j}}=\frac{R}{\sin \beta_{j}}=\frac{\left\|O Q_{1}\right\|}{\sin \gamma_{j}}
$$

式中, $O O_{1}$ 表示从时刻 $t-\tau_{j}$ 到 $t$, 铣刀中心的位移

$$
\left\|O O_{1}\right\|=f_{z} \frac{\tau_{j}}{T}
$$

且有

$$
\alpha_{j}= \begin{cases}\phi_{1}-\phi_{j} & \phi_{\mathrm{s}} \leqslant \phi_{j} \leqslant \frac{\pi}{2} \\ \phi_{j}-\phi_{1} & \frac{\pi}{2} \leqslant \phi_{j} \leqslant \phi_{\mathrm{e}}\end{cases}
$$

式中, $\phi_{\mathrm{s}}$ 和 $\phi_{\mathrm{e}}$ 分别等于齿 $j$ 过点 $Q_{\mathrm{s}}$ 和 $Q_{\mathrm{e}}$ 时的方位 角, 即槽铣时的切入和切出角。又有

$$
\beta_{j}= \begin{cases}\phi_{j}+\frac{\pi}{2} & \phi_{\mathrm{s}} \leqslant \phi_{j} \leqslant \frac{\pi}{2} \\ \frac{3 \pi}{2}-\phi_{j} & \frac{\pi}{2} \leqslant \phi_{j} \leqslant \phi_{\mathrm{e}}\end{cases}
$$




$$
\gamma_{j}= \begin{cases}\frac{\pi}{2}-\phi_{1} & \phi_{\mathrm{s}} \leqslant \phi_{j} \leqslant \frac{\pi}{2} \\ \phi_{1}-\frac{\pi}{2} & \frac{\pi}{2} \leqslant \phi_{j} \leqslant \phi_{\mathrm{e}}\end{cases}
$$

将式(13) (15)代入式(11)并用三角公式进行化简, 得

$$
\left\|O Q_{1}\right\|=R \cos \alpha_{j}-f_{\mathrm{z}} \frac{\tau_{j}}{T} \sin \phi_{j} \quad \phi_{\mathrm{s}} \leqslant \phi_{j} \leqslant \phi_{\mathrm{e}}
$$

将式(10)代入式(13), 整理后得

$$
\tau_{j}= \begin{cases}T\left(1-\frac{\alpha_{j}}{\phi_{\mathrm{p}}}\right) & \phi_{\mathrm{s}} \leqslant \phi_{j} \leqslant \frac{\pi}{2} \\ T\left(1+\frac{\alpha}{\phi_{\mathrm{p}}}\right) & \frac{\pi}{2} \leqslant \phi_{j} \leqslant \phi_{\mathrm{e}}\end{cases}
$$

将式(16)、(17)代入式(7), 整理后得

$$
h_{s, j}= \begin{cases}R\left(1-\cos \alpha_{j}\right)+f_{\mathrm{z}}\left(1-\frac{\alpha_{j}}{\phi_{\mathrm{p}}}\right) \sin \phi_{j} & \phi_{\mathrm{s}} \leqslant \phi_{j} \leqslant \frac{\pi}{2} \\ R\left(1-\cos \alpha_{j}\right)+f_{\mathrm{z}}\left(1+\frac{\alpha_{j}}{\phi_{\mathrm{p}}}\right) \sin \phi_{j} & \frac{\pi}{2} \leqslant \phi_{j} \leqslant \phi_{\mathrm{e}}\end{cases}
$$

根据图 1, 式(3)中的动态铣屑厚度可改为

$$
h_{\mathrm{d}, j}=\left[\begin{array}{ll}
\sin \phi_{j} & \cos \phi_{j}
\end{array}\right]\left[\begin{array}{l}
x_{j}(t)-x_{j}\left(t-\tau_{j}\right) \\
y_{j}(t)-y_{j}\left(t-\tau_{j}\right)
\end{array}\right]
$$

式(17) (19)即为时变迟滞参数、名义厚度和动态厚 度所满足的数学关系式, 其中的未知参数只有一个 $\alpha_{j}$ 。接下来分别采用数值方法和近似解析法求 取 $\alpha_{j}$, 进而得到铣削厚度。

\section{2 迟滞参数和铣屑厚度建模}

将式(12)、(14)、(17)分别代入式(11), 整理后 得

$$
\sin \alpha_{j}= \begin{cases}\frac{f_{\mathrm{z}}}{R}\left(1-\frac{\alpha_{j}}{\phi_{\mathrm{p}}}\right) \cos \phi_{j} & \phi_{\mathrm{s}} \leqslant \phi_{j} \leqslant \frac{\pi}{2} \\ -\frac{f_{\mathrm{z}}}{R}\left(1+\frac{\alpha_{j}}{\phi_{\mathrm{p}}}\right) \cos \phi_{j} & \frac{\pi}{2} \leqslant \phi_{j} \leqslant \phi_{\mathrm{e}}\end{cases}
$$

式(20)是以 $\alpha_{j}$ 为未知数的超越方程, 只能通过作图 法、近似解析法或数值方法求解。

图 3a、3b 分别显示了刀齿 $j$ 进入和离开切削区 域的情况, 刀齿只有处于切削区内才会产生铣屑和 铣屑力。从图 3 和图 2 可以看出, 从刀齿 $j$ 经过切 入点 $Q_{\mathrm{s}}$ 开始直到切出点 $Q_{\mathrm{e}}$ 为止, $O_{1} Q_{1}$ 和 $O Q_{j}$ 始终
相交, 构成夹角 $\alpha_{j}$ 。当 $\phi_{j}$ 从 $\phi_{\mathrm{s}}$ 增加到 $\pi / 2, \alpha_{j}$ 单调 减小到 0 ; 当 $\phi_{j}$ 从 $\pi / 2$ 增加到 $\phi_{\mathrm{e}}, \alpha_{j}$ 从 0 单调增加。 换句话说, 刀齿 $j$ 进入切削区域之后, $\alpha_{j}$ 与 $\phi_{j}$ 存在 函数关系。

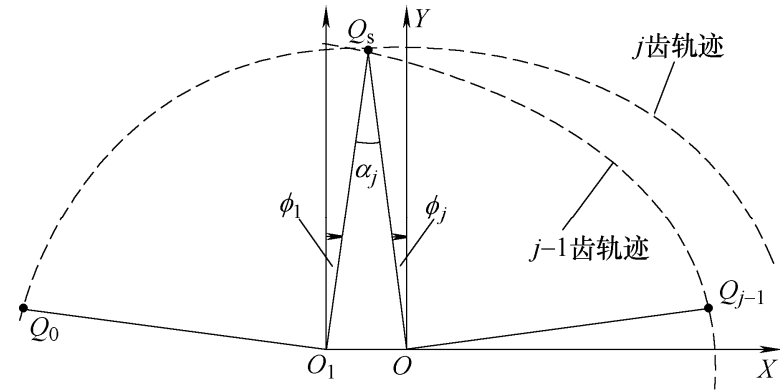

(a) 逆铣加工刀齿切入状态

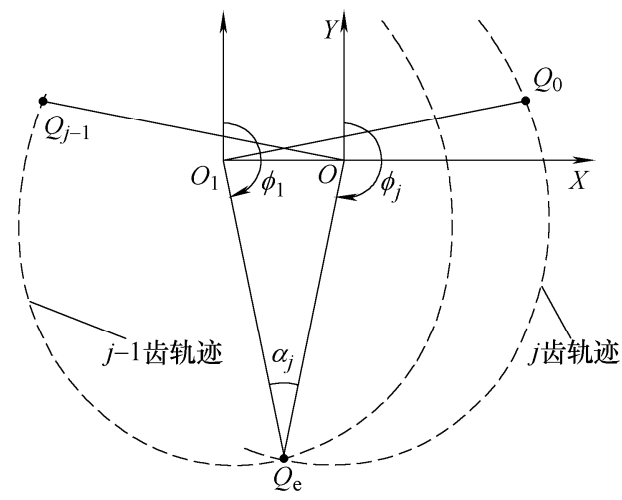

(b) 顺铣加工刀齿切出状态

图 3 切削区域的下限及上限

\section{1 数值方法}

要取得刀齿在任意方位角 $\phi_{j}$ 时的 $\alpha_{j}$ 值, 可以通 过牛顿-拉夫森等迭代算法求解式(20)。但是, 刀齿 每转到一个新的位置, 已经求解得到的结果并不能 用于后续求解, 需要对方程进行新的迭代搜索。无 疑, 需要求解的刀齿位置越多, 迭代求解在整个铣 削过程分析中消耗的计算成本就越多。

如果求解 $\alpha_{j}$ 方程的时候既能利用已有结果又能 避免循环迭代, 将大大改善数值计算的效率。注意 到 $\alpha_{j}$ 与 $\phi_{j}$ 存在函数关系, 在式(20)左右两边对 $\phi_{j}$ 求 导, 得

$$
\frac{\mathrm{d} \alpha_{j}}{\mathrm{~d} \phi_{j}} \cos \alpha_{j}=\left\{\begin{array}{l}
\frac{f_{\mathrm{z}} \sin \phi_{j}\left(\frac{\alpha_{j}}{\phi_{\mathrm{p}}}-1\right)-}{\frac{f_{\mathrm{z}} \cos \phi_{j}}{R \phi_{\mathrm{p}}} \frac{\mathrm{d} \alpha_{j}}{\mathrm{~d} \phi_{j}} \quad \phi_{\mathrm{s}} \leqslant \phi_{j} \leqslant \frac{\pi}{2}} \\
\frac{f_{\mathrm{z}} \sin \phi_{j}\left(1+\frac{\alpha}{\phi_{\mathrm{p}}}\right)-}{2} \\
\frac{f_{\mathrm{z}} \cos \phi_{j}}{R \phi_{\mathrm{p}}} \frac{\mathrm{d} \alpha_{j}}{\mathrm{~d} \phi_{j}} \quad \frac{\pi}{2} \leqslant \phi_{j} \leqslant \phi_{\mathrm{e}}
\end{array}\right.
$$


移项整理后, 得

$$
\frac{\mathrm{d} \alpha_{j}}{\mathrm{~d} \phi_{j}}= \begin{cases}\frac{\frac{f_{\mathrm{z}}}{R} \sin \phi_{j}\left(\frac{\alpha_{j}}{\phi_{\mathrm{p}}}-1\right)}{\cos \alpha_{j}+\frac{f_{\mathrm{z}}}{R \phi_{\mathrm{p}}} \cos \phi_{j}} & \phi_{\mathrm{s}} \leqslant \phi_{j} \leqslant \frac{\pi}{2} \\ \frac{f_{\mathrm{z}}}{R} \sin \phi_{j}\left(1+\frac{\alpha_{j}}{\phi_{\mathrm{p}}}\right) & \frac{\pi}{2} \leqslant \phi_{j} \leqslant \phi_{\mathrm{e}}\end{cases}
$$

式(22)是 $\alpha_{j}$ 的微分方程, 将其记为

$$
\frac{\mathrm{d} \alpha_{j}}{\mathrm{~d} \phi_{j}}=f\left(\phi_{j}, \alpha_{j}\right)
$$

于是, 可以利用求解常微分初值问题的数值方法得 到 $\alpha_{j}$, 例如下面所示的欧拉法或隆格-库塔法

$$
\alpha_{j, n+1}=\alpha_{j, n}+f\left(\phi_{j, n}, \alpha_{j, n}\right) \Delta \phi
$$

式中, $\phi_{j, n}$ 和 $\alpha_{j, n}$ 分别表示第 $n$ 个步长处的值; $\Delta \phi$ 表 示铣刀单位转角。

$$
\left\{\begin{array}{l}
K_{1}=f\left(\phi_{j, n}, \alpha_{j, n}\right) \\
K_{2}=f\left(\phi_{j, n}+\frac{\Delta \phi}{2}, \alpha_{j, n}+\frac{\Delta \phi}{2} K_{1}\right) \\
K_{3}=f\left(\phi_{j, n}+\frac{\Delta \phi}{2}, \alpha_{j, n}+\frac{\Delta \phi}{2} K_{2}\right) \\
K_{4}=f\left(\phi_{j, n}+\Delta \phi, \alpha_{j, n}+\Delta \phi K_{3}\right) \\
\alpha_{j, n+1}=\alpha_{j, n}+\frac{\Delta \phi}{6}\left(K_{1}+K_{2}+K_{3}+K_{4}\right)
\end{array}\right.
$$

从图 3a 可知, 逆铣刀齿刚进入切削区时满足

$$
\alpha_{j}=-2 \phi_{\mathrm{s}}
$$

将上式代入式(20), 得

$$
\sin \phi_{\mathrm{s}}+\frac{f_{\mathrm{z}} \phi_{\mathrm{s}}}{R \phi_{\mathrm{p}}}=-\frac{f_{\mathrm{z}}}{2 R}
$$

同样该式也无法求出解析解, 可以通过牛顿-拉夫森 法解出。逆铣加工的切出角取决于刀具半径和径向 切入深度 $a_{\mathrm{e}}$

$$
\phi_{\mathrm{e}}=\arccos \left(1-\frac{a_{\mathrm{e}}}{R}\right)
$$

对于顺铣, 切入角取决于刀具半径和径向切入深度

$$
\phi_{\mathrm{s}}=\pi-\arccos \left(1-\frac{a_{\mathrm{e}}}{R}\right)
$$

根据图 $3 b$ 可知，刀齿刚离开切削区时有

$$
\alpha_{j}=2\left(\phi_{\mathrm{e}}-\pi\right)
$$

将上式代入式(20), 得

$$
\frac{f_{\mathrm{z}}\left(\pi-\phi_{\mathrm{e}}\right)}{R \phi_{\mathrm{p}}}-\sin \phi_{\mathrm{e}}=\frac{f_{\mathrm{z}}}{2 R}
$$

现将求取铣削厚度的数值算法的步骤总结如下。

(1) 确定微分方程式(22)的初始条件 $\left(\phi_{j, 0}, \alpha_{j, 0}\right)$, 其中 $\phi_{j, 0}=\phi_{\mathrm{s}}$ 。如果是逆铣或槽铣, 使用迭代搜索解 出式(27)中的切入角 $\phi_{\mathrm{s}}$, 然后代入式(26)得到 $\alpha_{j, 0}$; 如果是顺铣, $\phi_{\mathrm{s}}$ 由式(29)得到, 然后将其代入式(20) 并使用迭代搜索求出 $\alpha_{j, 0}$ 。

(2) 确定微分方程(22)的自变量取值上限。如果 是逆铣, 切出角 $\phi_{\mathrm{e}}$ 由式(28)得到; 如果是顺铣或槽 铣，使用迭代搜索解出式(31)中的切入角 $\phi_{\mathrm{e}}$ 。

(3) 利用式(24)或(25)等其他数值方法求解方程 (22), 得到 $\alpha_{j, n}$ 。

(4) 将 $\alpha_{j, n}$ 代入式(17) (19), 得到铣削厚度。

从上述步骤可知, 本文所提数值方法在求解铣 削厚度的整个过程中, 最多只需要两次迭代搜索算 法用于确定定解条件。

\section{2 解析法}

如果 $\alpha_{j}$ 的值很小, 用线性函数 $y=\alpha_{j}$ 近似正弦函 数 $y=\sin \alpha_{j}$ 导致的误差常常可以忽略。例如, 当 $\alpha_{j}$ 小于 $\pi / 10$, 线性近似后的误差不超过 $1.7 \%$ 。因此, 将式(20)等号左边的 $\sin \alpha_{j}$ 替换为 $\alpha_{j}$, 整理后得到

$$
\alpha_{j}= \begin{cases}\frac{\phi_{\mathrm{p}} f_{\mathrm{z}} \cos \phi_{j}}{\phi_{\mathrm{p}} R+f_{\mathrm{z}} \cos \phi_{j}} & \phi_{\mathrm{s}} \leqslant \phi_{j} \leqslant \frac{\pi}{2} \\ -\frac{\phi_{\mathrm{p}} f_{\mathrm{z}} \cos \phi_{j}}{\phi_{\mathrm{p}} R+f_{\mathrm{z}} \cos \phi_{j}} & \frac{\pi}{2} \leqslant \phi_{j} \leqslant \phi_{\mathrm{e}}\end{cases}
$$

将式(32)代入式(17), 整理得到时变迟滞参数的表达 式为

$$
\tau_{j}=\frac{T}{\frac{f_{\mathrm{z}}}{\phi_{\mathrm{p}} R} \cos \phi_{j}+1} \quad \phi_{\mathrm{s}} \leqslant \phi_{j} \leqslant \phi_{\mathrm{e}}
$$

同理, 假设如果 $\alpha_{j}$ 的值很小, 则式(18)中 $\alpha_{j}$ 的余弦 函数可以用 2 次函数近似, 得到

$$
h_{s, j}= \begin{cases}\frac{R}{2} \alpha_{j}^{2}+f_{\mathrm{z}}\left(1-\frac{\alpha_{j}}{\phi_{\mathrm{p}}}\right) \sin \phi_{j} & \phi_{\mathrm{s}} \leqslant \phi_{j} \leqslant \frac{\pi}{2} \\ \frac{R}{2} \alpha_{j}^{2}+f_{\mathrm{z}}\left(1+\frac{\alpha_{j}}{\phi_{\mathrm{p}}}\right) \sin \phi_{j} & \frac{\pi}{2} \leqslant \phi_{j} \leqslant \phi_{\mathrm{e}}\end{cases}
$$

将式(32)代入上式，整理得到名义铣屑厚度为

$$
h_{s, j}=\frac{R}{2}\left(\frac{\phi_{\mathrm{p}} f_{\mathrm{z}} \cos \phi_{j}}{f_{\mathrm{z}} \cos \phi_{j}+\phi_{\mathrm{p}} R}\right)^{2}+\frac{\phi_{\mathrm{p}} R f_{\mathrm{z}} \sin \phi_{j}}{f_{\mathrm{z}} \cos \phi_{j}+\phi_{\mathrm{p}} R}
$$

式(35)与 FAASSEN 等 ${ }^{[19]}$ 采用不同推导过程得到的 模型类似。由式(19)、式(33)和式(35)可得铣削厚度 近似解析模型为 


$$
\begin{gathered}
h_{j}=h_{\mathrm{s}, j}+h_{\mathrm{d}, j}=\left[\begin{array}{cc}
\sin \phi_{j} & \cos \phi_{j}
\end{array}\right] \times \\
{\left[\begin{array}{c}
\mu_{j} f_{\mathrm{z}}+x_{j}(t)-x_{j}\left(t-\mu_{j} T\right) \\
\frac{\cos \phi_{j}}{2 R}\left(\mu_{j} f_{\mathrm{z}}\right)^{2}+y_{j}(t)-y_{j}\left(t-\mu_{j} T\right)
\end{array}\right]} \\
\mu_{j}=\frac{1}{\frac{f_{\mathrm{z}}}{\phi_{\mathrm{p}} R} \cos \phi_{j}+1}
\end{gathered}
$$

式(36)与式(38)所示的圆形轨迹铣屑厚度模型 在形式上非常类似, 区别在于式(36)含有时变因子 $\mu_{j}$ 及式(38)忽略了进给运动在 $Y$ 向对名义厚度的影响。

$$
h_{j}=\left[\begin{array}{ll}
\sin \phi_{j} & \cos \phi_{j}
\end{array}\right]\left[\begin{array}{c}
f_{\mathrm{z}}+x_{j}(t)-x_{j}(t-T) \\
y_{j}(t)-y_{j}(t-T)
\end{array}\right]
$$

将式(26)代入式(32), 整理后得到逆铣或槽铣切 入角为

$$
\phi_{\mathrm{s}}=\frac{-\phi_{\mathrm{p}} f_{\mathrm{z}}}{2\left(f_{\mathrm{z}}+\phi_{\mathrm{p}} R\right)}
$$

将式(30)代入式(32), 整理后得到顺铣或槽铣切出角 为

$$
\phi_{\mathrm{e}}=\pi+\frac{\phi_{\mathrm{p}} f_{\mathrm{z}}}{2\left(\phi_{\mathrm{p}} R-f_{\mathrm{z}}\right)}
$$

\section{3 仿真对比及分析}

因为铣屑厚度包含名义厚度和动态厚度, 其中 名义厚度体现的是铣削进给产生的铣屑, 而动态厚 度是反映的是铣削过程中的振动对铣屑厚度的影 响。名义厚度只需要已知铣削加工条件即可确定, 动态厚度则需求解系统动力学方程式(4)才能得到。 下面首先通过和其他名义厚度模型对比, 然后进行 铣削过程仿真, 验证本文所提方法的有效性。

\section{1 铣屑厚度仿真}

加工条件为槽铣、 $f_{\mathrm{z}}=0.2 \mathrm{~mm} /$ 齿, 刀具参数 $N_{t}=4$ 、 $R=5 \mathrm{~mm}$, 不同名义铣屑厚度模型的仿真结果如图 4 所示: 实线和带星号的实线图形分别表示采用数值 法式(25)和近似解析式(35)得到铣屑厚度; 虚线图形 以及带空心三角、圆圈和矩形标识的虚线图形分 别表示采用圆形轨迹模型、MARTELLOTTI ${ }^{[1]}$ 、 LI 等 ${ }^{[13]}$ 和 FAASSEN 等 ${ }^{[19]}$ 的模型计算得到的铣屑厚 度。FAASSEN 和 LI 提出的名义厚度表达式分别为

$$
h_{\mathrm{s}, j}=R-R \cos \left(\frac{\phi_{\mathrm{p}} f_{\mathrm{z}} \cos \phi_{j}}{f_{\mathrm{z}} \cos \phi_{j}+\phi_{\mathrm{p}} R}\right)+\frac{\phi_{\mathrm{p}} f_{\mathrm{z}} R \sin \phi_{j}}{f_{\mathrm{z}} \cos \phi_{j}+\phi_{\mathrm{p}} R}
$$

$$
\begin{gathered}
h_{\mathrm{s}, j}=R\left(1-\left(1-\frac{2 \phi_{\mathrm{p}} f_{\mathrm{z}} \sin \phi_{j}}{\phi_{\mathrm{p}} R+f_{\mathrm{z}} \cos \phi_{j}}-\right.\right. \\
\left.\left.\frac{f_{\mathrm{z}}^{2} \phi_{\mathrm{p}}^{2} \cos 2 \phi_{j}}{\left(\phi_{\mathrm{p}} R+f_{\mathrm{z}} \cos \phi_{j}\right)^{2}}+\frac{f_{\mathrm{z}}^{3} \phi_{\mathrm{p}}^{3} \sin \phi_{j} \cos ^{2} \phi_{j}}{\left(\phi_{\mathrm{p}} R+f_{\mathrm{z}} \cos \phi_{j}\right)^{3}}\right)^{\frac{1}{2}}\right)
\end{gathered}
$$

从图 4 可见, 各图形重叠到了一起, 难以分辨, 说明各模型计算结果基本一致。为了更清楚地区分 图 4 中各种模型的仿真结果, 以步长为 $\phi_{\mathrm{p}} / 200$ 的数 值解为参照, 图 5 显示了各解析模型与数值解之间 的相对误差。可以看到圆形轨迹模型的精确程度最 低, 且总的来说低估了铣屑厚度; 在切入和切出角 附近, 文献[1]的摆线轨迹模型存在 $\pm 3 \%$ 的相对误差, 而其他几种模型的误差均低于 $0.1 \%$ 。

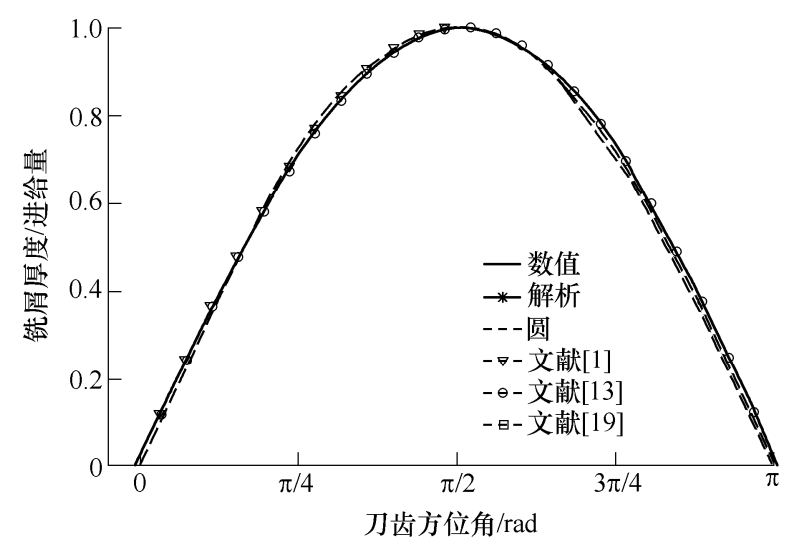

图 4 不同名义厚度模型仿真结果

( $R=5 \mathrm{~mm}, N_{t}=4, f_{\mathrm{z}}=0.2 \mathrm{~mm} /$ 齿, 槽铣)

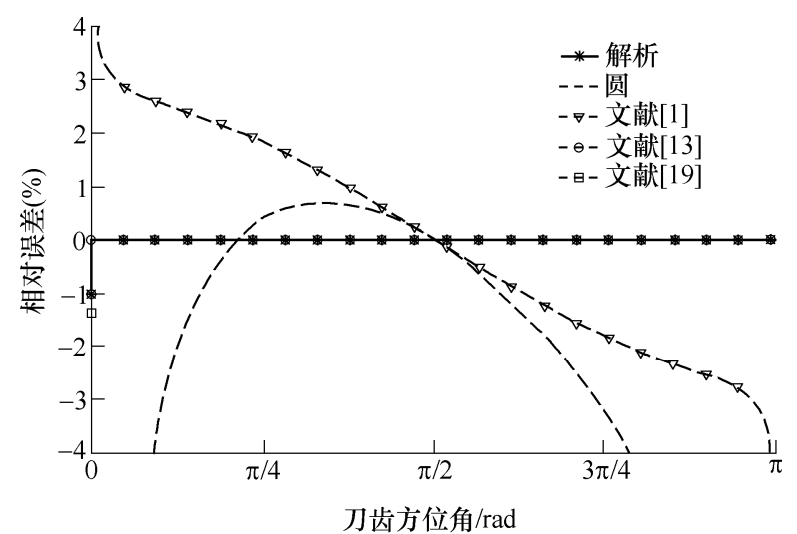

图 5 不同解析模型的误差

$\left(R=5 \mathrm{~mm}, N_{t}=4, f_{\mathrm{z}}=0.2 \mathrm{~mm} /\right.$ 齿, 槽铣)

由于式(1)和(2)将 $\tau$ 当作常数处理, 如果进给量 $f_{\mathrm{z}}$ 增大, 文献[1]的模型和圆形轨迹模型的误差将会 更大更明显。图 6 所示为 $f_{\mathrm{z}} / R=0.4$ 时的仿真结果, 可以看出文献[1]的模型和圆形轨迹模型的计算结 果均出现了巨大的偏差, 而其他几种摆线轨迹解析 模型仍与数值解总体上吻合得很好。 


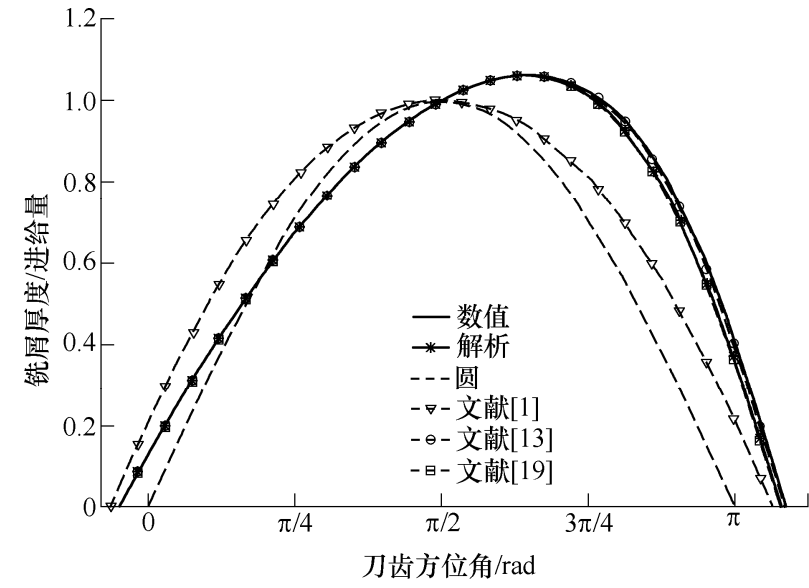

图 6 不同名义铣屑厚度模型仿真结果

( $R=5 \mathrm{~mm}, N_{t}=4, f_{\mathrm{z}}=2 \mathrm{~mm} /$ 齿, 槽铣)

图 7 所示为刀齿数改为 8 时的仿真结果。可以 看出, 当方位角小于 $\pi / 2$ 时, 除了圆形轨迹模型和 文献[1]模型之外, 其他模型结果仍然一致; 但是, 当方位角大于 $\pi / 2$ 之后, 各模型开始出现较大偏差, 并且用数值法计算的铣屑厚度逐渐趋于无穷大，而 各解析模型仍然给出了收敛答案。

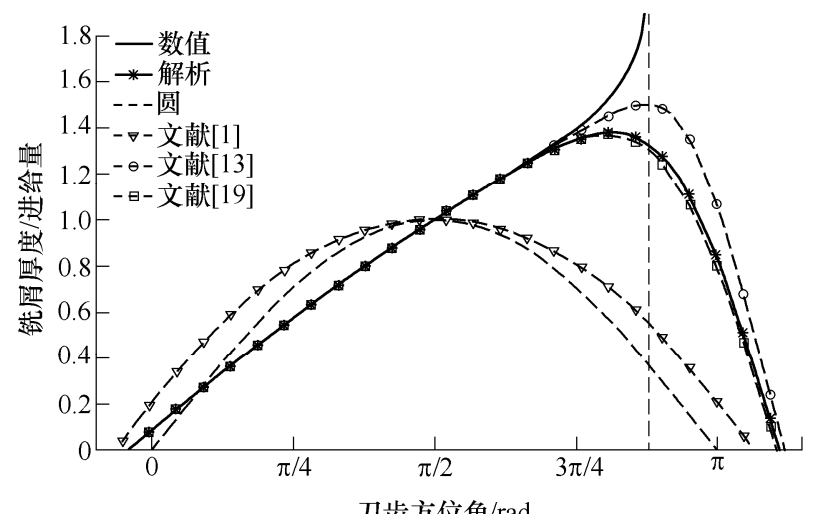

图 7 不同名义铣屑厚度模型仿真结果

$\left(R=5 \mathrm{~mm}, N_{t}=8, f_{\mathrm{z}}=2 \mathrm{~mm} /\right.$ 齿, 槽铣)

从图 2 和图 3 所示的刀齿 $j$ 的轨迹与前一刀齿 的轨迹之间的几何关系可知, 在一个刀齿切削周期 内, 夹角 $\alpha_{j}$ 是刀齿角位移 $\phi_{j}$ 的连续函数, 并且在 $\phi_{j}$ 等于 $\pi / 2$ 时取极小值 0 , 在 $\phi_{j}$ 等于 0 和 $\pi$ 时有两个 极大值; 而 $\pi / 2<\phi_{j}$ 时, 式(22)等号右边部分的分母 有可能等于 0 , 则 $\alpha_{j}$ 将出现无穷间断点, 且只有 $f_{\mathrm{z}} /\left(R \phi_{\mathrm{p}}\right)$ 足够大的时候该断点才会发生。因此, 从数 学的角度看, 若铣刀半径不变, 增加进给量或齿数 都有可能导致铣屑厚度趋于无穷大。上述各近似解 析模型均基于 $\alpha_{j}$ 足够小的假设, 对刀齿运动方程进 行化简, 因此不能反映出 $\alpha_{j}$ 可能存在间断点的情况, 从而给出了错误的结果。

图 4 7 的仿真对比结果表明, 对于逆铣或槽铣 刀齿方位角小于 $\pi / 2$ 的名义铣屑厚度, 本文及文献
[12-13]和文献[19]给出的基于摆线轨迹的近似解析 模型均能给出和数值解一致的结果，对于顺铣或槽 铣刀齿方位角大于 $\pi / 2$ 的铣屑厚度, 随着进给量或 齿数的增加, 近似解析模型的误差越来越大。文献 [12-13]的模型误差相对最小，但是表达式最复杂。 本文解析模型误差略小于文献[19]的模型, 且表达 式相对简单。

\section{2 铁削过程动力学仿真}

将式(5)代入式(4), 得到铣削过程动力学方程为

$$
\boldsymbol{M} \ddot{\boldsymbol{q}}+\boldsymbol{C} \dot{\boldsymbol{q}}+\boldsymbol{K q}=
$$

$$
\sum_{j=1}^{N_{t}} \int_{0}^{a_{\mathrm{p}}} g\left(\phi_{j}\right)\left(\boldsymbol{A}\left(\phi_{j}\right)\left(h_{\mathrm{s}, j}+h_{\mathrm{d}, j}\right)+\boldsymbol{B}\left(\phi_{j}\right)\right) \mathrm{d} z
$$

式中, 矩阵函数 $\boldsymbol{A}$ 和 $\boldsymbol{B}$ 分别为

$$
\begin{aligned}
\boldsymbol{A}\left(\phi_{j}\right) & =\left[\begin{array}{cc}
-\cos \phi_{j} & -\sin \phi_{j} \\
\sin \phi_{j} & -\cos \phi_{j}
\end{array}\right]\left[\begin{array}{l}
K_{\mathrm{t}, \mathrm{c}} \\
K_{\mathrm{n}, \mathrm{c}}
\end{array}\right] \\
\boldsymbol{B}\left(\phi_{j}\right) & =\left[\begin{array}{cc}
-\cos \phi_{j} & -\sin \phi_{j} \\
\sin \phi_{j} & -\cos \phi_{j}
\end{array}\right]\left[\begin{array}{l}
K_{\mathrm{t}, \mathrm{e}} \\
K_{\mathrm{n}, \mathrm{e}}
\end{array}\right]
\end{aligned}
$$

由于动态铣屑厚度同时包含当前和过去的刀具动态 位移向量, 可知式(43)属于时变迟滞微分方程。式(43) 只能通过数值方法进行求解。方程中的迟滞项采用 如下线性插值处理

$$
\left\{\begin{array}{l}
\boldsymbol{q}\left(t-\tau_{j}(t)\right)=a_{j, n} \boldsymbol{q}_{n-m_{j, n}}+b_{j, n} \boldsymbol{q}_{n-m_{j, n}+1} \\
a_{j, n}=\frac{\tau_{j}(t)+\left(n-m_{j, n}+1\right) \Delta t-t}{\Delta t} \\
b_{j, n}=\frac{t-\tau_{j}(t)-\left(n-m_{j, n}\right) \Delta t}{\Delta t}
\end{array}\right.
$$

式中 $\boldsymbol{q}_{n}=\boldsymbol{q}(n \Delta t), \Delta t$ 为时间步; $m_{j, n}=$ floor $\left(\tau_{j}\left(t_{n}\right) / \Delta t+1 / 2\right)$, floor 为向下取整函数;

下面以文献[21]中的铣削系统参数为例, 计算 不同加工条件下的铣削力和刀具动态位移。系统模 态对称, 频率 $319.38 \mathrm{~Hz}$, 阻尼比 $1.96 \%$, 刚度 $2.16 \times 10^{7} \mathrm{~N} / \mathrm{m} ; R=10 \mathrm{~mm}, N_{t}=4$, 螺旋角 $30^{\circ}$; $K_{\mathrm{t}, \mathrm{c}}=804.3 \mathrm{MPa}, K_{\mathrm{n}, \mathrm{c}}=331 \mathrm{MPa}, K_{\mathrm{t}, \mathrm{e}}=K_{\mathrm{n}, \mathrm{e}}=0$ 。

图 8 所示为采用摆线轨迹铣屑模型的仿真结 果, 仿真步长 $\Delta t=10^{-5} \mathrm{~s}$, 铣削加工条件为: 顺铣, $a_{\mathrm{p}}=9 \mathrm{~mm}, a_{\mathrm{e}}=1 \mathrm{~mm}, \omega=10^{4} \mathrm{r} / \mathrm{min}$, 图 $8 \mathrm{a}$ 中 $f_{\mathrm{z}}=0.2 \mathrm{~mm} /$ 齿, 图 $8 \mathrm{~b}$ 中 $f_{\mathrm{z}}=2 \mathrm{~mm} /$ 齿。可以看到: 图 $8 \mathrm{a}$ 中模型 处于稳定铣削状态, $Y$ 向铣削力和动态位移幅值分 别为约 $200 \mathrm{~N}$ 和 $4.5 \mu \mathrm{m}$; 图 $8 \mathrm{~b}$ 中模型处于不稳定铣 削状态, 铣削力在 $0.15 \mathrm{~s}$ 就超过 $4000 \mathrm{~N}$, 动态位移 的幅值增长到 $0.2 \mathrm{~mm}$ 以上。

图 9 所示为采用圆形轨迹铣屑模型的仿真结 果, 仿真参数与图 8 相同。从图 9 可见, 虽然进给 

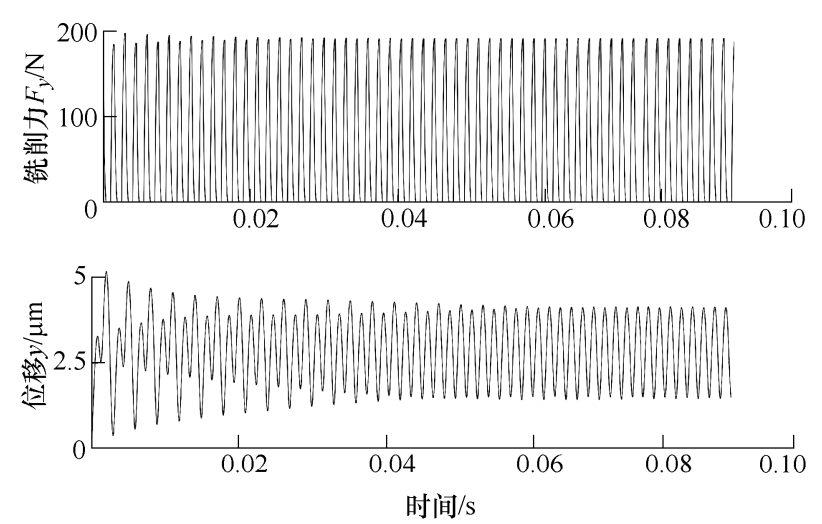

(a) $f_{\mathrm{z}}=0.2 \mathrm{~mm} /$ 齿, $a_{\mathrm{p}}=9 \mathrm{~mm}, a_{\mathrm{e}}=1 \mathrm{~mm}, \omega=10^{4} \mathrm{r} / \mathrm{min}$
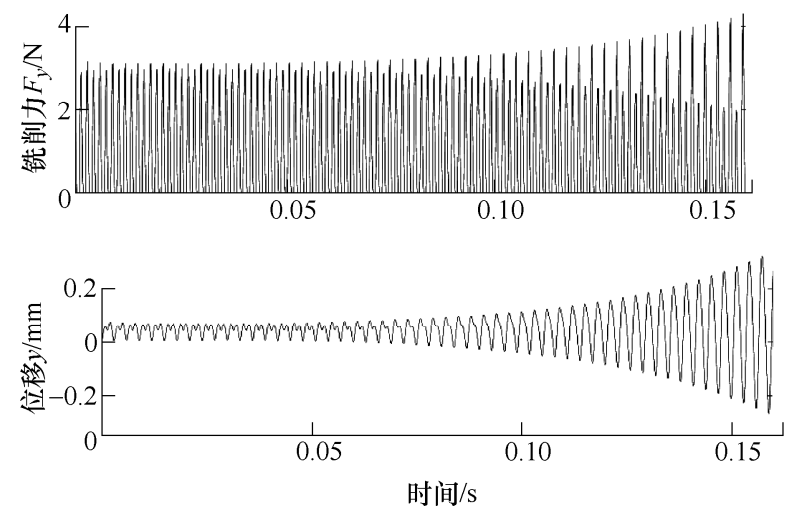

(b) $f_{\mathrm{z}}=2 \mathrm{~mm} /$ 齿, $a_{\mathrm{p}}=9 \mathrm{~mm}, a_{\mathrm{e}}=1 \mathrm{~mm}, \omega=10^{4} \mathrm{r} / \mathrm{min}$

图 8 基于摆线轨迹铣屑模型的仿真结果
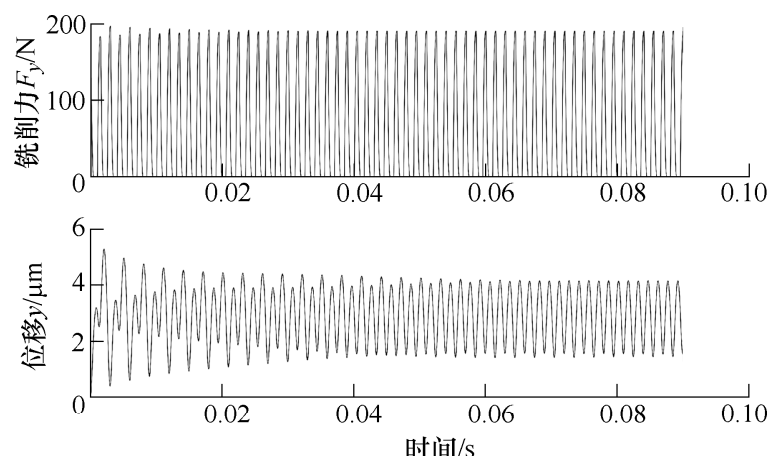

(a) $f_{\mathrm{z}}=0.2 \mathrm{~mm} /$ 齿, $a_{\mathrm{p}}=9 \mathrm{~mm}, a_{\mathrm{e}}=1 \mathrm{~mm}, \omega=10^{4} \mathrm{r} / \mathrm{min}$
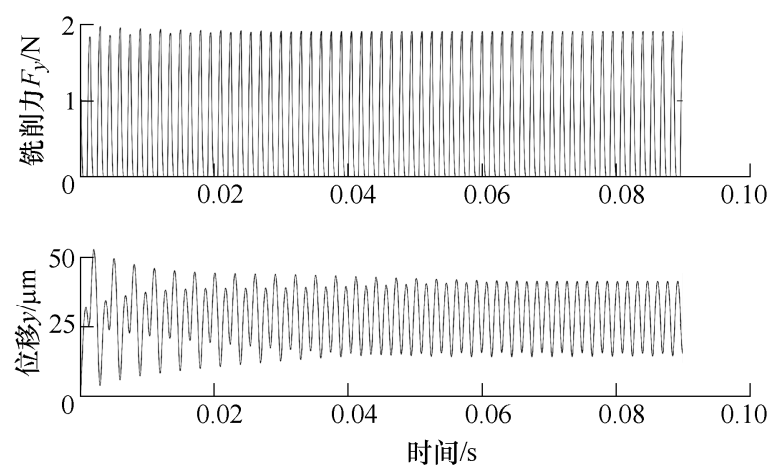

(b) $f_{\mathrm{z}}=2 \mathrm{~mm} /$ 齿, $a_{\mathrm{p}}=9 \mathrm{~mm}, a_{\mathrm{e}}=1 \mathrm{~mm}, \omega=10^{4} \mathrm{r} / \mathrm{min}$

图 9 基于圆形轨迹铣屑模型的仿真结果

量改变之后, 图 $9 \mathrm{a}$ 和 $9 \mathrm{~b}$ 的 $Y$ 向铣削力和动态位移 的最大幅值不同, 但是都处于稳定铣削状态。

对比图 8 和图 9 可知, 当 $f_{\mathrm{z}}$ 较小时基于两种铣 屑模型的铣削过程仿真得到了一致的结果。但是,

基于摆线轨迹铣屑厚度建立的铣削过程模型采用不 同的进给量 $f_{\mathrm{z}}$ 将表现出不同的动态特性; $f_{\mathrm{z}}$ 对采用 圆形轨迹铣屑厚度的铣削过程的动态特性没有任何 影响, 因而给出错误的结果。

为了说明本文所提求解未变形铣削厚度的数值 方法能够提高整个铣削过程动力学仿真的效率, 分 别采用 “迭代法” 和本文所提方法进行仿真。仿真 软件系统为 Matlab 9.0。在仿真过程中, 除了计算 未变形铣削厚度部分的代码, “迭代法” 采用系统内 置非线性方程求解器 “fzero” (求解误差设为 $10^{-3}$, 其余属性均为默认值), 本文方法采用式(22)和(25) 之外, 其余仿真程序内容完全相同并且在同一台电 脑上运行(AMD A10-6800K4.4 GHz, 8 GBRAM)。

表 1 列出了铣削过程进行到 $0.1 \mathrm{~s}$ 时的 $Y$ 向铣削 力、刀具动态位移和仿真程序消耗的时长, 铣削加 工条件与图 $8 \mathrm{a}$ 相同。可见步长 $\Delta t \leqslant 10^{-5} \mathrm{~s}$ 时两种方 法铣削力和刀具动态位移都收玫且结果是一致的, 但迭代法仿真耗时约为本文所提方法的 25 倍。

表 1 不同方法仿真结果

\begin{tabular}{lcccc}
\hline \multirow{2}{*}{ 结果 } & \multicolumn{4}{c}{ 步长 $\Delta t / \mathrm{s}$} \\
\cline { 2 - 5 } & $1 \times 10^{-4}$ & $2 \times 10^{-5}$ & $1 \times 10^{-5}$ & $5 \times 10^{-6}$ \\
\hline 迭代法 $F_{y} / \mathrm{N}$ & 169.7066 & 189.8731 & 200.1100 & 199.9122 \\
本文方法 $F_{y} / \mathrm{N}$ & 170.0616 & 190.1379 & 200.5285 & 200.2511 \\
迭代法 $y / \mu \mathrm{m}$ & 1.5574 & 1.4255 & 1.5363 & 1.5457 \\
本文方法 $y / \mu \mathrm{m}$ & 1.5090 & 1.4222 & 1.5512 & 1.5696 \\
迭代法耗时 $/ \mathrm{s}$ & 2.6359 & 14.0705 & 28.9830 & 58.8222 \\
本文方法耗时/s & 0.1019 & 0.5533 & 1.1275 & 3.3787 \\
耗时比 & 25.8675 & 25.4301 & 25.7055 & 17.4097 \\
\hline
\end{tabular}

\section{4 结论}

（1）本文所提解析法可以提供和其他基于摆线 轨迹的解析模型相当的精度, 且数学表达式更简单, 本文解析模型同时包含名义铣屑厚度和动态铣屑 厚度, 在形式上与经典的圆形轨迹铣屑模型相似, 因此有利于完全替代圆形轨迹铣屑模型运用于铣 削过程建模。值得注意的是, 逆铣或槽铣刀齿方位 角小于 $\pi / 2$ 的时候, 解析法始终可以给出与数值法 一致的结果; 而对于对于顺铣或槽铣刀齿方位角大 于 $\pi / 2$ 的情况, 随着进给量或齿数的增加, 解析 法的误差越来越大。其他摆线轨迹解析模型也存在 这个问题。

(2) 采用本文所提数值法不需要在每一个时间 步长都进行迭代寻根运算求解铣屑厚度, 可以将计 算铣屑厚度的步骤嵌入到整个铣削过程动力学方程 求解过程中, 并且可以采用相同的步长和数值方法, 
进一步提高分析效率。大部分基于时域解的铣削过 程稳定性分析需要针对不同主轴转速求解铣削动力 学方程, 计算非常耗时。因此, 在铣削稳定性分析 时采用本文所提求解未变形铣屑厚度的方法也可以 提高稳定性分析的精度和效率。

\section{参 考 文 献}

[1] MARTELLOTTI M E. An analysis of the milling process[J]. Transactions of the ASME, 1941, 63(8): 677-700.

[2] SRIDHAR R, HOHN R E, LONG G W. A General formulation of the milling process equation: Contribution to machine tool chatter research-5[J]. Journal of Engineering for Industry, 1968, 90(2): 317-324.

[3] SABBERWAL A J P. Cutting forces in down milling[J]. International Journal of Machine Tool Design and Research, 1962, 2(1): 27-41.

[4] TLUSTY J, ISMAIL F. Basic non-linearity in machining chatter[J]. CIRP Annals, 1981，30(1): 299-304.

[5] SMITH S, TLUSTY J. An overview of modeling and simulation of the milling process[J]. Journal of Engineering for Industry， 1991， 113(2): 169-175.

[6] BUDAK E, ALTINTAS Y, ARMAREGO E J A. Prediction of milling force coefficients from orthogonal cutting data[J]. Journal of Manufacturing Science \& Engineering, 1996, 118(2): 216-224.

[7] BUDAK E. Analytical models for high performance milling. Part I: Cutting forces, structural deformations and tolerance integrity[J]. International Journal of Machine Tools and Manufacture, 2006, 46(12): 1478-1488.

[8] MONTGOMERY D, ALTINTAS Y. Mechanism of cutting force and surface generation in dynamic milling[J]. Journal of Engineering for Industry, 1991, 113(2): 160-168.

[9] ALTINTAS Y, MONTGOMERY D. Dynamic peripheral milling of flexible structures[J]. Journal of Engineering for Industry, 1992, 114(2): 137-145.

[10] CAMPOMANES M L, ALTINTAS Y. An improved time domain simulation for dynamic milling at small radial immersions[J]. Journal of Manufacturing Science and Engineering, 2003, 125(3): 416-422.

[11] SCHMITZ T L, COUEY J, MARSH E, et al. Runout effects in milling: Surface finish, surface location error, and stability[J]. International Journal of Machine Tools and Manufacture, 2007, 47(5): 841-851.

[12] LI H Z, LI X P. A numerical study of the effects of cutter runout on milling process geometry based on true tooth trajectory $[\mathrm{J}]$. The International Journal of Advanced Manufacturing Technology, 2005, 25(5-6): 435-443.

[13] LI H Z, LIU K, LI X P. A new method for determining the undeformed chip thickness in milling[J]. Journal of Materials Processing Technology, 2001, 113(1): 378-384.

[14] KUMANCHIK L M, SCHMITZ T L. Improved analytical chip thickness model for milling $[\mathrm{J}]$. Precision Engineering, 2007, 31(3): 317-324.

[15] 间雪, 陶华, 蔡晋, 等. 基于真实刀刃轨迹的立铣刀切 削厚度模型 $[\mathrm{J}]$. 机械工程学报, 2011，47(1): 182-186. YAN Xue, TAO Hua, CAI Jin, et al. Model of the instantaneous undeformed chip thickness in milling based on real tooth trajectory[J]. Journal of Mechanical Engineering, 2011, 47(1): 182-186.

[16] LONG X H, BALACHANDRAN B. Milling model with variable time delay[C]//ASME 2004 International Mechanical Engineering Congress and Exposition. American Society of Mechanical Engineers, 2004 : 933-940.

[17] LONG X H, BALACHANDRAN B, MANN B P. Dynamics of milling processes with variable time delays[J]. Nonlinear Dynamics, 2007, 47(1): 49-63.

[18] LONG X, INSPERGER T, BALACHANDRAN B. Systems with periodic coefficients and periodically varying delays : Semidiscretization-based stability analysis[M]//Delay Differential Equations. New York: Springer, 2009.

[19] FAASSEN R, VAN DE WOUW N, NIJMEIJER H, et al. An improved tool path model including periodic delay for chatter prediction in milling[J]. Journal of Computational and Nonlinear Dynamics, 2007, 2(2): 167-179.

[20] SONG G, LI J, SUN J. Approach for modeling accurate undeformed chip thickness in milling operation[J]. The International Journal of Advanced Manufacturing Technology, 2013, 68(5-8): 1429-1439.

[21] ZATARAIN M, MUNOA J, PEIGNÉ G, et al. Analysis of the influence of mill helix angle on chatter stability[J]. CIRP Annals-Manufacturing Technology，2006， 55(1): 365-368.

作者简介: 窦炜, 男, 1980 年出生, 博士研究生。主要研究方向为机床 动力学和机械可靠性。

E-mail: Mech_2010_dou@163.com

何晓聪(通信作者), 男, 1955 年出生, 博士, 教授, 博士研究生导师。 主要研究方向为薄板材料连接、机械动力学和系统可靠性理论。

E-mail: xiaocong_he@126.com 